\title{
Spatial Homogenization Algorithm for Bridging Disparities in Scale between the Fire and
}

\section{Solid Domains}

\author{
Paul A. Beata and Ann E. Jeffers ${ }^{*}$ \\ University of Michigan, Ann Arbor, MI USA \\ "Corresponding Author, Tel.: +1 734 7636832, Fax: +1 734764 4292, Email: jffrs@ umich.edu
}

Abstract: The analysis of structures exposed to non-uniform heating from localized fires is a challenging task due to the spatially varying boundary conditions and the differences in scale between the fire simulation and solid heat transfer model. This paper presents a spatial homogenization algorithm for capturing non-uniform boundary conditions from a highresolution fire simulation in a low-resolution finite element heat transfer model of a structure. The homogenization algorithm uses numerical integration by the trapezoid rule to calculate the equivalent thermal flux vector in the finite element heat transfer model for a spatially varying surface flux. The proposed method is compared to other approximating techniques, including averaging, sampling, and least squares methods, for a $2 \mathrm{D}$ heat transfer problem. The results demonstrate that the proposed homogenization algorithm converges rapidly due to the energyequivalent representation of the thermal boundary condition. The homogenization algorithm is then implemented in a 3D heat transfer model that uses macro-level plate elements. For an application involving a horizontal plate exposed to a localized fire, the model is shown to converge to the results obtained by a solid finite element model. The homogenization algorithm combined with the plate heat transfer element proves to be an accurate and highly efficient means for analyzing structures with spatially varying thermal boundary conditions calculated by computational fluid dynamics.

Keywords: fire simulation, heat transfer, finite element analysis, numerical methods, structures 


\section{Introduction}

Current methods for the fire-resistant design of structures emphasize the design for postflashover fire conditions and commonly use parametric fire models such as those found in Eurocode 1 [1]. Post-flashover fire models assume that the gas temperature is uniform within the compartment. Although intending to represent a worst-case structurally significant fire, postflashover fire models are limited to relatively small compartments with regular geometries. In large open spaces, fires tend to burn locally and oftentimes will travel across the floor plate and even spread across floors [2]. Under these conditions, the fire behavior can be rather complex and may be more accurately represented by a computational fluid dynamics (CFD) model.

CFD models such as Fire Dynamics Simulator (FDS) [3] are well-established and provide an accurate representation of natural fire events. However, challenges arise when coupling the CFD fire model to a solid heat transfer model due to incompatibilities in mesh and disparities in scale between the fire and solid domains. Figure 1 illustrates the disparities in spatial and temporal scales for a one-way coupled system. Note that an extreme case of a fire impinging on the structure is shown for contrast. It can be seen that the fire simulation requires a time step and spatial resolution that is considerably smaller than is needed in the structural analysis due to fire dynamics being a "fast" physics. Yu and Jeffers [4] demonstrated that a time-averaged subcycling algorithm can overcome the differences in temporal scales between the fire simulation and solid heat transfer model in an accurate and efficient manner. However, the difference in spatial scale becomes particularly important when simulating the $3 \mathrm{D}$ temperature gradients in the structure. 


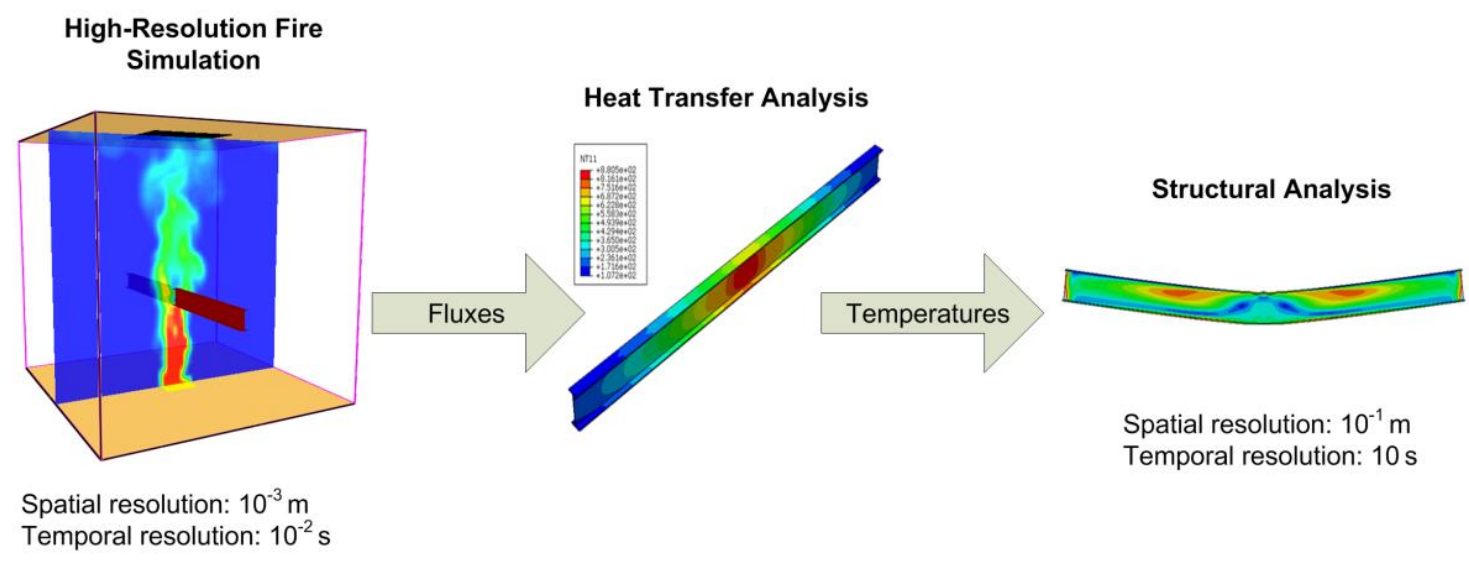

Figure 1: Differences in scale for a sequentially coupled analysis (the extreme case of a fire impinging on a structure is shown)

There have been a few efforts in recent years to couple a CFD fire model to a structural model in order to study structural response under natural fire effects. The NIST investigations into the World Trade Center collapses employed high-fidelity CFD fire models in conjunction with macro finite element structural models [5]. In the NIST investigations, the boundary conditions were expressed as a locally uniform gas temperature that was calculated by the CFD analysis. In a similar vein, Tondini et al. [6-7] established a framework to couple a CFD fire model to a structural model based on a locally uniform gas temperature. Wickstrom et al. [8] demonstrated that the boundary condition at the fire-structure interface could be expressed in terms of adiabatic surface temperature, which decouples the fire exposure from the structural surface temperature. These approaches are most suitable for situations in which the fire heats the structure by remote radiation or by heat transfer associated with an optically thick gas, in which case the fire exposure is relatively uniform over the structure's surface. Temperature gradients along the lengths of members can be captured by discretizing the structural members into more elements [6]. However, temperature gradients along the lengths of members were not the focus of prior studies. 
Non-uniform heating in general is known to have a significant effect on the structural response [9-12]. Additionally, it has been shown that localized heating associated with a fire impinging on a structure can be detrimental to the structural performance because it can produce structural effects (e.g., local buckling) that are not seen when the structure is heated uniformly [13]. It has not yet been determined whether fluctuations in boundary conditions due to a real fire impinging on a structure play a significant role in the thermo-mechanical response of the structure. It is clear that unprotected structures will be more sensitive to such localized effects, but even for unprotected members the effects have not been quantified. To this end, Chen et al. [14] established a coupling interface between a CFD fire model and finite element heat transfer model of a structure based on convection between the structure and a gas of non-uniform temperature. While able to simulate non-uniform heating along the lengths of members, the work by Chen et al. used 3D solid elements in the finite element model, resulting in a structural model that required excessive computational expense due to the fact that a fine mesh was needed to capture the cross-section response. Thus, although the CFD analysis continues to drive the total computing time in coupled fire-structure models, it is apparent that 3D solid finite elements are unnecessarily inefficient for thin-walled structures, leaving room for further improvement.

To improve computational efficiency in the thermo-mechanical analysis of beams, plates, and shells, recent efforts by Jeffers et al. [15-17] have led to a class of macro heat transfer elements that can simulate the response of non-uniformly heated structures in an accurate and computationally efficient manner. Specifically, beam, plate, and shell heat transfer elements (Fig. 2) were formulated for calculating the $3 \mathrm{D}$ thermal response of non-uniformly heated structures. A combination of finite element and control volume methods was used in the element formulations to solve the 3D conduction heat transfer equations in an accurate and efficient 
manner. The elements have fiber or layered discretizations to account for large temperature gradients over the cross-section or through the thickness, respectively. Temperatures along the length or in plane are approximated by linear or quadratic shape functions. One advantage to the use of macro heat transfer elements is that the elements facilitate the transfer of data from a thermal analysis to a structural analysis because both models can have the same mesh.

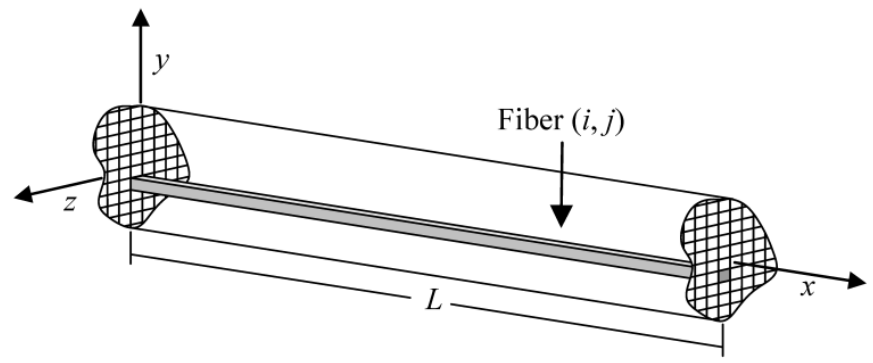

(a)

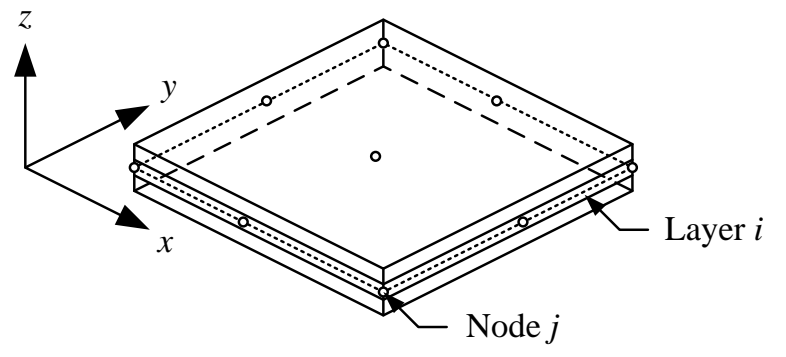

(b)

Figure 2: Macro heat transfer elements: (a) fiber heat transfer element [15], (b) plate/shell heat transfer element [16]

The macro elements have been proven to provide computational savings that are more than an order of magnitude over 3D solid elements. In the case of the plate heat transfer element, for example, it was shown in a 3D verification study that the element provided the same level of accuracy as a 3D solid model and required 1.3 to $2.6 \mathrm{~s}$ to perform an analysis that required 830.7 seconds for a 3D solid model [16]. The computational savings are related to the significant reduction in the number of temperature degrees of freedom that are needed in the heat transfer model. The macro elements also allow for a much coarser mesh along the length or in plane in 
the heat transfer analysis. In the analysis of a $4 \mathrm{~m}$ steel beam exposed to a localized fire, it was shown that an element size of $50 \mathrm{~cm}$ to $100 \mathrm{~cm}$ in length provided a prediction of the thermomechanical response that was comparable to a 3D solid model [15]. In the analysis of a $6 \mathrm{~m} \times 6$ $\mathrm{m}$ concrete slab exposed to a localized fire, temperatures were accurate for an element that was $37.5 \mathrm{~cm}$ in length [18].

This paper concerns a spatial homogenization algorithm for overcoming the differences in spatial scales between the fire simulation and solid heat transfer model, with an emphasis on conduction heat transfer evaluated by macro finite elements. For macro elements in particular, it is necessary to consider the case in which the CFD grid is significantly finer than the finite element grid and requires homogenization of the data from the CFD analysis. A numerical integration scheme based on the trapezoid rule is employed in the calculation of the equivalent nodal flux vector for the heat transfer finite element. The proposed homogenization algorithm is compared to other methods for representing the spatially varying boundary condition, including averaging, sampling, and least squares methods. Following the $2 \mathrm{D}$ verification study, this paper considers a 3D application of a horizontal plate exposed to localized fire, in which the fire exposure is simulated by CFD and the solid heat transfer analysis is conducted using the plate heat transfer element in [16].

\section{Governing Equations}

The equations governing conduction heat transfer by finite element analysis are expressed as

$$
[C]\{\dot{T}\}+[K]\{T\}=\{R\}
$$


where $\{\boldsymbol{T}\}$ is the field variable (temperature), $[C]$ is the heat capacity matrix, $[K]$ is the thermal conductivity matrix, $\{R\}$ is the vector of thermal loads, which includes heat flux, radiation, and convection boundary conditions [19]. For the applications considered here, it is assumed that incident heat fluxes $q$ " are calculated by CFD analysis and losses by convection and radiation are separately accounted for. Thus, the homogenization algorithm is presented for a flux boundary condition, although the methodology can readily be extended to convection and radiation boundary conditions. It is presently assumed for combustible solids that the pyrolysis gases flow outward from the surface, in which case there is no forcing term related to mass conservation that is passed into the solid model from the CFD analysis.

The vector of heat fluxes is obtained by summing the heat flux vectors for each of the elements in the structure. For an incident heat flux boundary condition, the vector $\{r\}$ for an element is expressed as

$$
\{r\}=\int_{S}\lfloor N\rfloor^{T} q^{\prime \prime} d S
$$

where $\lfloor N\rfloor$ is a matrix containing the element's shape functions and $q^{\prime \prime}$ is the heat flux acting over surface $S$. Under natural fire conditions, $q^{\prime \prime}$ may vary greatly over the surface $S$ due to turbulent flow as illustrated in Fig. 3a. Thus, the objective is to compute Eq. (2) as an equivalent nodal flux vector (Fig. 3b) that appropriately accounts for the non-uniformity in the boundary condition. This can be done by (i) replacing $q^{\prime \prime}$ with a function that approximates the randomly varying boundary condition with a smooth (e.g., linear) function, or (ii) expressing Eq. (2) in a discrete form that preserves the non-uniformity in $q^{\prime \prime}$. The former approach might be chosen for convenience due to compatibility with the standard finite element formulation, whereas the latter approach may be more precise, as shown in this paper. Several methods were investigated for 
representing the non-uniform boundary condition for a $2 \mathrm{D}$ planar problem. Of the methods investigated for the planar model, only the highest performing approach was used in the extension to $3 \mathrm{D}$.

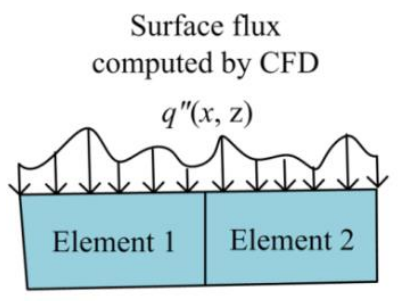

(a)

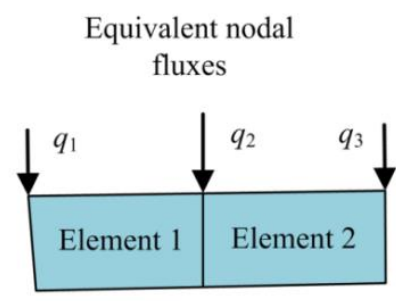

(b)

Figure 3: 2D elements exposed to non-uniform surface flux: (a) non-uniform flux, and (b) equivalent nodal fluxes

\subsection{D Formulation}

In each method explored in the current study, the goal was to approximate Eq. (2) given the randomly varying heat flux $q^{\prime \prime}$. For a 2D isoparametric element, Eq. (2) can be expressed as

$$
\{r\}=\int_{-1}^{+1}\lfloor N(\xi)\rfloor^{T} q^{\prime \prime}(\xi) t J d \xi
$$

where $\xi$ is the natural coordinate ranging from -1 to $1 ;\lfloor N\rfloor$ is an array containing the element shape functions, which are expressed in terms of $\xi$; $q^{\prime \prime}$ is the heat flux, which varies in $\xi$; $t$ is the thickness of the element; and $J$ is the determinant of the Jacobian matrix relating the natural coordinates $\xi \eta$ to the global $x y$ coordinates.

Four homogenization algorithms were investigated for handling a randomly distributed heat flux over the surface of an element, as illustrated in Fig. 4. The average value method applies a uniform heat flux equal to the average of the randomized fluxes over the surface of the element. The sampling method takes the flux values at the end points of the element and interpolates linearly between them. The least squares method fits the randomized flux data with a linear least squares approximation. The trapezoid method preserves the randomized flux data and 
uses the trapezoid rule to numerically evaluate the integral in Eq. (3). The averaging, sampling, and least squares methods aim to smooth the randomized flux with a linear function, enabling the Gaussian quadrature rule to be used for numerical integration, whereas the trapezoid method expresses Eq. (3) in a discrete form that captures the randomized flux exactly. It should be noted that the trapezoid rule is superior to Gaussian quadrature for the integration of a random function because Gaussian quadrature is best-suited for the integration of low-order polynomials. However, averaging, sampling, and least squares methods are convenient methods for smoothing the data from a CFD analysis. This paper considers the implications of smoothing the data vs. integrating the function in a more exact sense by studying the structural temperatures in realistic fire safety engineering applications.

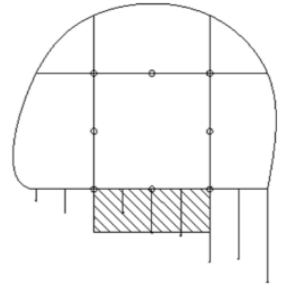

Average Value

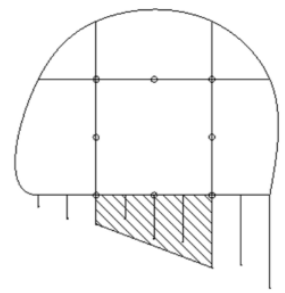

Sampling

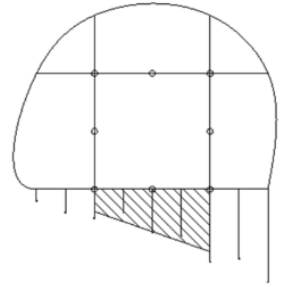

Least Squares

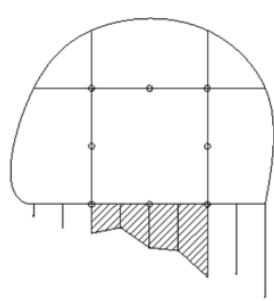

Trapezoid Rule

Figure 4: Overview of the four methods developed for 2D spatial homogenization

The integral in Eq. (3) was evaluated numerically in each of the four methods. Numerical integration of the element's flux vector $\{r\}$ was accomplished using Gaussian quadrature in the averaging, sampling, and least squares methods, whereby the integral in Eq. (3) is expressed as

$$
\{r\} \approx \sum_{i=1}^{n} W_{i}\left\lfloor N\left(\xi_{i}\right)\right\rfloor^{T} q^{\prime \prime}\left(\xi_{i}\right) t J
$$

In Gaussian quadrature, $W_{i}$ represents the weighting constant associated with the sampling point $\xi_{i}$, which lies in the domain $[-1,1]$ along the isoparametric element's edge. The heat flux $q^{\prime \prime}(\xi)$ 
is evaluated at Gauss point $\xi_{i}$ based on the homogenization method that is used. The summation is carried out over $n$, the number of sampling points based on the quadrature rule that is employed.

The trapezoid rule was explored as an alternative to the methods based on Gaussian quadrature. This method was intentionally selected because it uses all of the data associated with the element in calculating the equivalent nodal flux vector (as opposed to sampling at the Gauss points). In this manner, the contribution of each measured heat flux from the fire simulation is accounted for as opposed to a sampling technique which could fail to catch peaks in the heat flux. By integrating the random flux over the surface, the nodal flux vector that is calculated by the trapezoid rule is equivalent in energy to the random heat flux that exists at the element's surface.

Figure 5 shows the application of the trapezoid rule in 2D for a non-uniform heat flux $q^{\prime \prime}(\xi)$ at the $\eta=1$ edge of the element. The data points refer to sensor data from the CFD fire simulation for incident heat fluxes. As shown in the figure, five heat flux data points are used to approximate the integral with four trapezoidal segments. In the general case for $2 \mathrm{D}$ elements, $n+1$ points are used for $n$ trapezoidal segments along a given element's edge. When using data from CFD, the width of each trapezoid segment is dependent on the spacing of the sensors in the fire simulation (i.e., the fidelity of the CFD mesh). It is assumed that the number of data points between end nodes is large in comparison to the number of nodes (a reflection of the differences in scale between the fire and solid heat transfer models). The integral in Eq. (3) is evaluated by applying the trapezoid rule using incident heat flux data from the fire simulation. For $n+1$ data points, 


$$
\{r\} \approx\left\lfloor N\left(\xi_{0}\right)\right\rfloor^{T} q^{\prime \prime}\left(\xi_{0}\right) t J+2 \sum_{i=1}^{n-1}\left\lfloor N\left(\xi_{i}\right)\right\rfloor^{T} q^{\prime \prime}\left(\xi_{i}\right) t J+\left\lfloor N\left(\xi_{n}\right)\right\rfloor^{T} q^{\prime \prime}\left(\xi_{n}\right) t J
$$

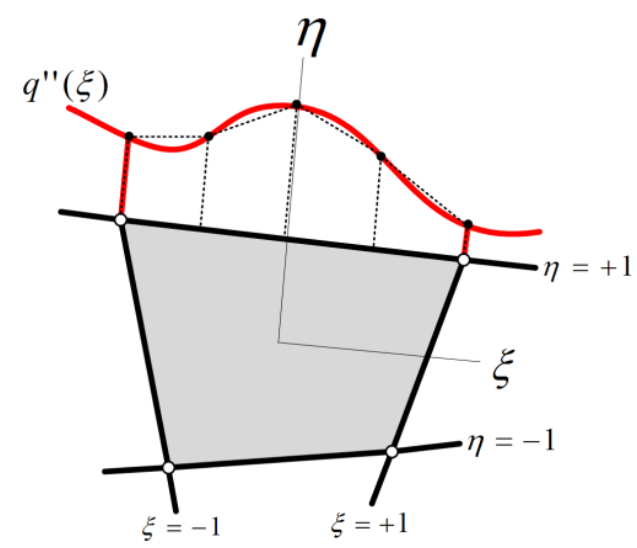

Figure 5: Isoparametric element with non-uniform boundary condition

\subsection{D Formulation}

The extension to $3 \mathrm{D}$ is essential for using the spatial homogenization algorithm in the calculation of equivalent nodal fluxes for non-uniform thermal boundary conditions applied over surfaces as opposed to edges of the element. Fig. 6a shows a spatially varying heat flux over the surface of a macro heat transfer element. As in the 2D case, equivalent nodal fluxes are to be calculated from the discrete data and applied at the nodes, as shown in Fig. 6b. The homogenization algorithm based on the trapezoid rule may be extended to a second dimension by simply applying the trapezoid rule to several slices of data in one dimension (say, along the $\xi$ direction) and then subsequently applying the trapezoid rule in the second dimension (along the $\eta$-direction). 


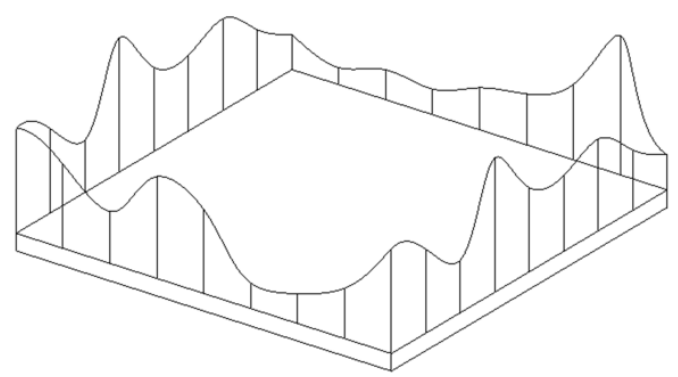

(a)

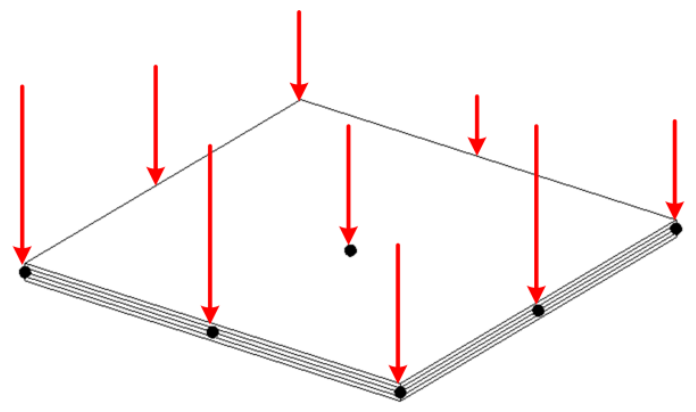

(b)

Figure 6: 3D shell heat transfer element with non-uniform thermal load

The heat flux vector of Eq. (3) can be re-written by adding another dimension to the expression of the heat flux vector:

$$
\{r\}=\int_{S}\lfloor N\rfloor^{T} q^{\prime \prime} d S=\int_{-1}^{+1} \int_{-1}^{+1}\lfloor N(\xi, \eta)\rfloor^{T} q^{\prime \prime}(\xi, \eta) J d \xi d \eta
$$

Note that the shape functions $N$, heat flux $q^{\prime \prime}$, and Jacobian $J$ are now functions of two coordinates, $\xi$ and $\eta$, representing the surface $S$ over which the heat flux is applied. The Jacobian is used to map between the real space and the natural coordinates of the isoparametric element, as shown in Fig 7. Note that the heat transfer finite element can have arbitrary geometry due to the isoparametric formulation. Thus, it is not necessary that the finite element grid overlay the CFD grid, provided that the CFD model can handle complex solid boundaries.

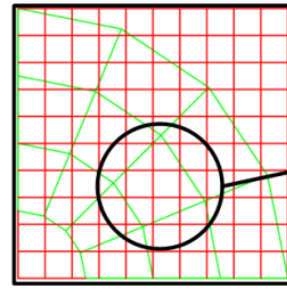

Irregular FEA Mesh on CFD Grid

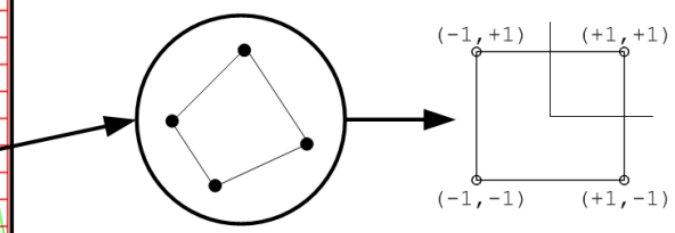

Single 4-node Element Mapped Isoparametric from the FEA Mesh Element $[-1,1]$

Figure 7: Mapping between real and natural coordinates 


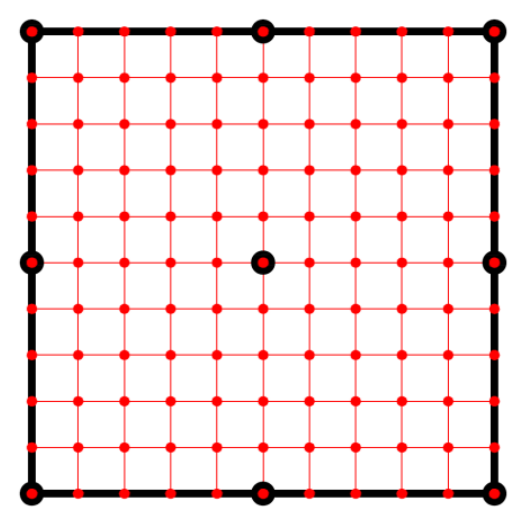

Figure 8: Flux data sensor mesh on a single element

For a rectangular element, heat fluxes are measured over a uniform grid as shown in Fig. 8. Equation (6) is used to calculate the heat flux vector for the element. First, integration is performed along the straight lines of the grid in one dimension, as shown in Fig. 9, resulting in $m+1$ integrals along the first dimension (say, in the $\xi$-direction as before). The integral at a particular line in natural coordinates along the element's surface will be denoted as integral $j$ for $j=0,1, \ldots, m$.

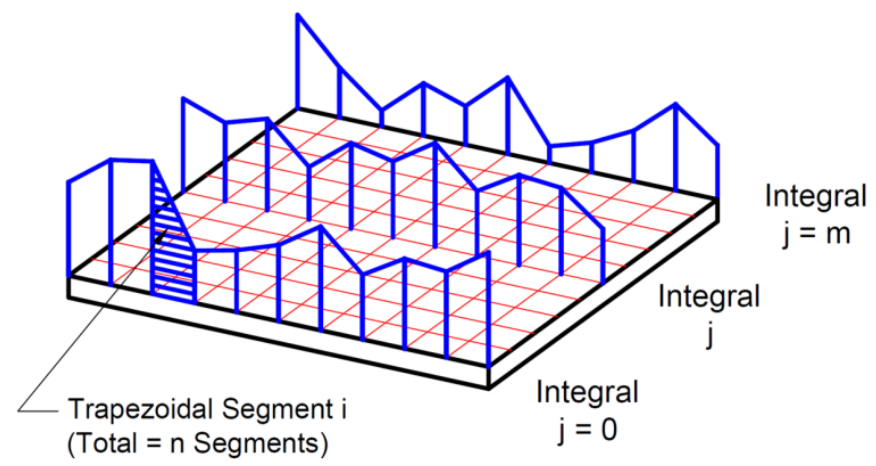

Figure 9: Integration over a surface using the trapezoid rule

From Eq. (6), it can be seen that each of the $j$ integrals results in an arraywith the same size as the number of nodes in the element. The vector representing slice $j$ is defined with a dummy variable $\{I\}^{(j)}$, where 


$$
\begin{gathered}
\{I\}^{(j)}=\left\lfloor N\left(\xi_{0}, \eta_{j}\right)\right\rfloor^{T} q^{\prime \prime}\left(\xi_{0}, \eta_{j}\right) J_{0, j}+2 \sum_{i=1}^{n-1}\left\lfloor N\left(\xi_{i}, \eta_{j}\right)\right\rfloor^{T} q^{\prime \prime}\left(\xi_{i}, \eta_{j}\right) J_{i, j} \\
+\left\lfloor N\left(\xi_{n}, \eta_{j}\right)\right\rfloor^{T} q^{\prime \prime}\left(\xi_{n}, \eta_{j}\right) J_{n, j}
\end{gathered}
$$

Note that $\{I\}^{(j)}$ is based on the 1D trapezoid rule for $\xi$ ranging from -1 to 1 and $\eta=\eta_{j}$. In total, there are $m$ vectors $\{I\}^{(j)}$ produced by integrating over the $j=0,1, \ldots, m$ slices of data.

The integration over the surface of the element is computed by applying the trapezoid rule to vectors $\{I\}^{(j)}$ for $\eta$ ranging for -1 to 1 , i.e.,

$$
\{r\} \approx \frac{1}{m}\left(\{I\}^{(0)}+2 \sum_{j=1}^{m-1}\{I\}^{(j)}+\{I\}^{(m)}\right)
$$

This integration method requires calculation of the element flux vector by the trapezoid rule using the number of data points available at the surface of each element. It is not required that the CFD grid matches the FEA grid, although compatibility between meshes facilitates the transfer of data between the two models. For the case in which the CFD grid does not match the FEA grid, integration points are defined on the element in isoparametric coordinates (e.g., trapezoid rules of order $h, h / 2$, and $h / 4$ are shown in Fig. 10). The heat fluxes from the CFD grid points that are adjacent to the integration point are interpolated linearly to get the heat flux data that is passed into the trapezoid rule, as shown in Fig. 10. 


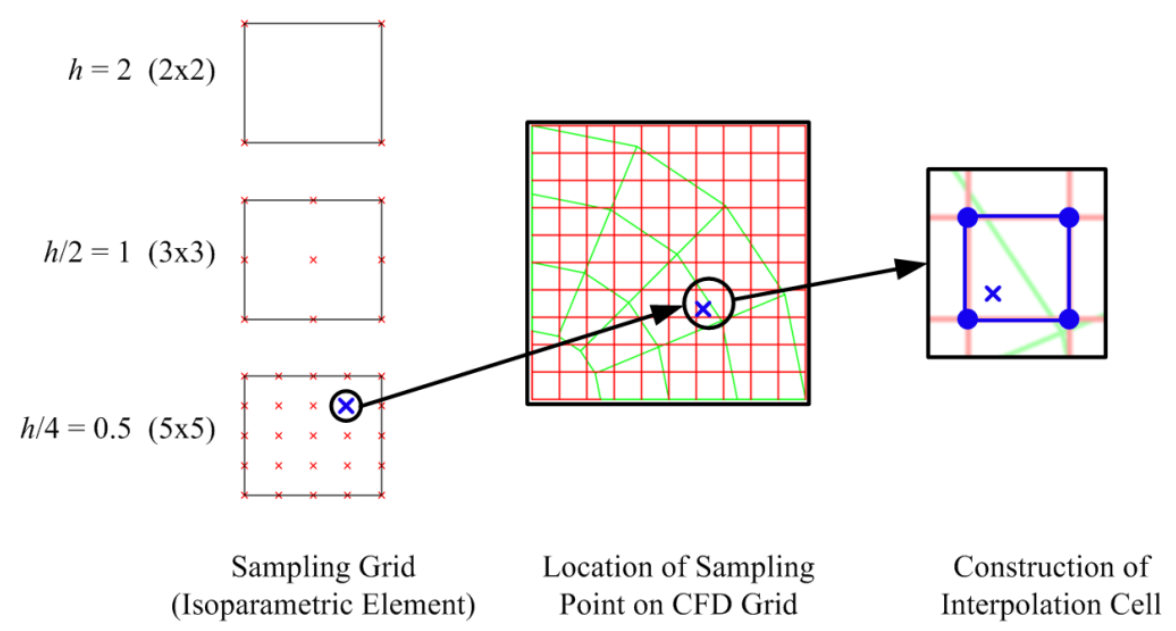

Figure 10: Visualization of the mapping process based on sampling scheme used

\section{Implementation}

With an efficient numerical method for calculating energy-equivalent nodal fluxes from spatially varying incident heat flux data obtained by a CFD fire simulation, the non-uniform thermal boundary conditions produced in a natural fire scenario can be passed into a finite element heat transfer analysis model. The averaging, sampling, and least squares methods were compared to the trapezoid method for spatial homogenization in two dimensions. The trapezoid method was extended for a 3D case and implemented in a macro heat transfer element [16] that has a coarse grid in relation to the CFD grid. It should be noted that the homogenization technique for handling non-uniform thermal boundary conditions is particularly useful for macro-level finite elements, which have a coarser element mesh in relation to the CFD grid.

This paper considers a sequentially coupled analysis in which the fire affects heat transfer to the structure but the structure does not affect the fire dynamics. A flow chart is provided in Fig. 11, which shows the transfer of data through the analysis. In Fig. 11, the disparities in spatial scale between the fire and solid domains are handled using a homogenization algorithm based on sampling, averaging, least squares, or trapezoid methods. To conduct the analysis, a CFD 
simulation is performed to compute the incident heat flux acting on the surfaces of the structure. The homogenization algorithm transforms large sets of data describing boundary conditions at a structure's surface into equivalent heat fluxes that act at the nodes of the finite element in the conduction heat transfer analysis. An input file is generated for the finite element heat transfer analysis, with the equivalent nodal heat fluxes specified as boundary conditions. The heat transfer model is then analyzed to determine the temperatures within the solid. For a two-way coupled system, surface fluxes and temperatures computed in the heat transfer analysis would be calculated at the CFD grid points by interpolation using the element's shape functions. The methodology presumes that the CFD grid is finer than the element mesh used in the solid heat transfer model, which is generally the case when macro heat transfer elements are used.

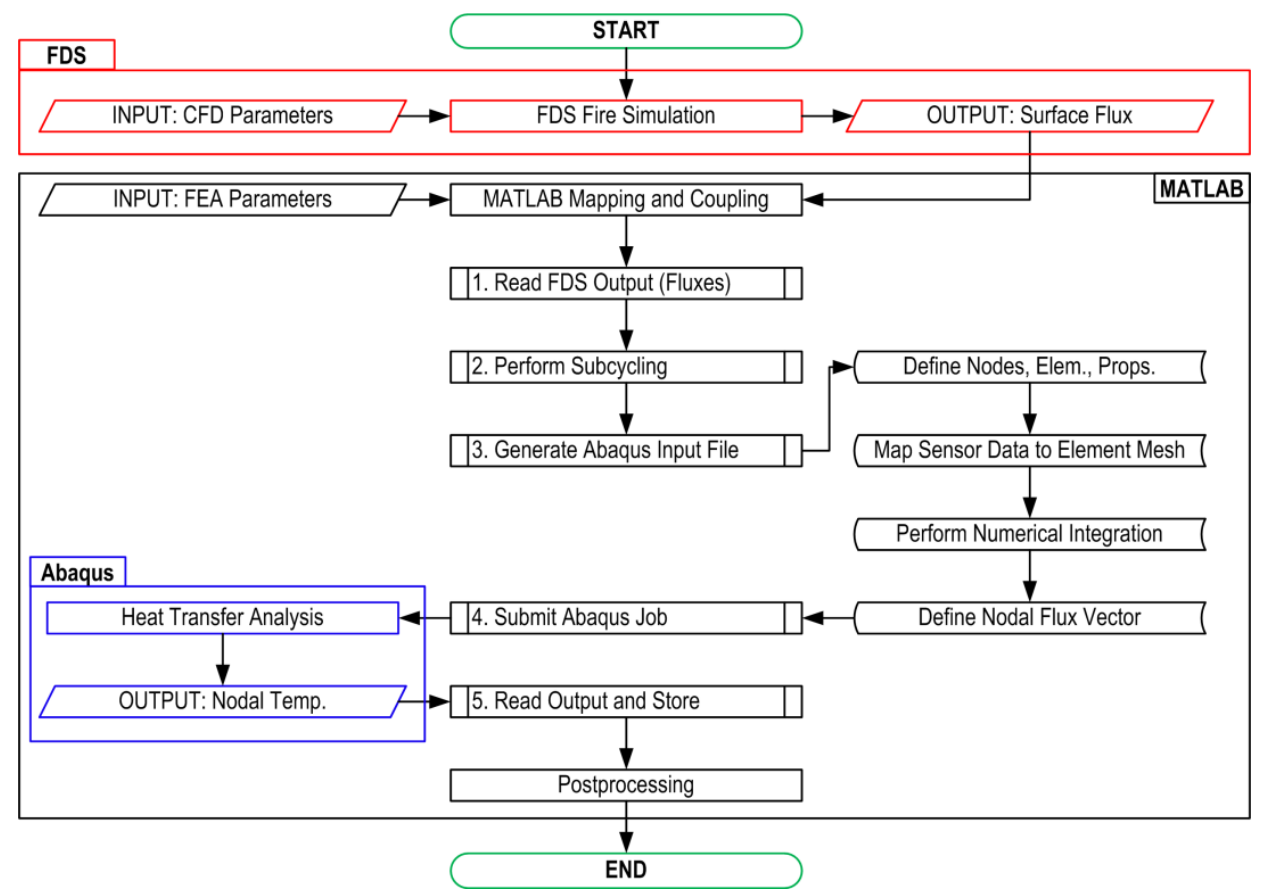

Figure 11: Flowchart for sequentially coupled analysis

\subsection{D Implementation}

A 2D application was considered for the evaluation of the homogenization algorithms based on averaging, sampling, least squares, and the trapezoid rule. In the $2 \mathrm{D}$ application, the 
conduction heat transfer to the solid was evaluated using eight-node (quadratic) solid elements with the equivalent nodal fluxes calculated by Eq. (4) for the averaging, sampling, and least squares methods, or Eq. (5) for the trapezoid method. On the heated surface, the boundary condition was expressed as a net heat flux, i.e.,

$$
q^{\prime \prime}=q_{n e t}^{\prime \prime}
$$

which was assumed to be constant in time so as to avoid challenges associated with differences in time scale that would otherwise occur in a coupled fire-structure simulation. To simplify the problem, a random heat flux was generated in MATLAB as opposed to performing a CFD fire simulation for the 2D analysis. It was also assumed that the heat flux variation out of plane was negligible such that the problem could be treated as $2 \mathrm{D}$. The $2 \mathrm{D}$ heat transfer analysis was performed in a special-purpose code that was written in MATLAB.

\subsection{D Implementation}

As will be shown in the following sections, the trapezoid method was found to exhibit superior performance over the averaging, sampling, and least squares methods. The algorithm based on the trapezoid rule was therefore extended to $3 \mathrm{D}$ heat transfer in Section 2.2 and implemented with the layered plate heat transfer element in Fig. 2b [16] for an application involving a plate exposed to a localized fire. In the plate heat transfer element, a heat flux boundary condition is applied to a layer at the top or bottom of the plate using Eq. (2). For homogenization of a spatially varying heat flux, the trapezoid rule is applied according to Eqs. (7)-(8). The present analysis considers a nine-node quadratic plate element, although the formulation in Section 2.2 is general and can be applied to any type and order of element. In the 3D case, the spatially varying heat flux was calculated by CFD analysis in Fire Dynamics 
Simulator (FDS). The boundary conditions are expressed in terms of an incident heat flux with losses to the surroundings by convection and radiation.

The 3D conduction heat transfer analysis was performed in Abaqus. A special purpose code was written in MATLAB to resolve the spatially varying surface fluxes into equivalent nodal fluxes according to the spatial homogenization algorithm in Eqs. (7)-(8). The plate heat transfer element was implemented in Abaqus as a user-defined element (i.e., UEL subroutine). The equivalent nodal fluxes were specified using the *DFLUX command in the input file after they were calculated in a preprocessing program in MATLAB (see flowchart in Fig. 11).

\section{Results}

\subsection{D Application}

The homogenization algorithms based on averaging, sampling, least squares, and the trapezoid rule were evaluated by considering a plate exposed to a random (i.e., non-uniform) net heat flux along one edge, as shown in Fig. 12. The average heat flux varied linearly from 10 $\mathrm{kW} / \mathrm{m}^{2}$ at the ends of the plate to $40 \mathrm{~kW} / \mathrm{m}^{2}$ at the center of the plate. About the mean, the heat flux followed a random distribution that was bounded by a range of $20 \mathrm{~kW} / \mathrm{m}^{2}$. The random heat flux can be seen in Fig. 12 along with the boundaries. The heat flux was assumed to be constant over the thickness of the plate (i.e., out of plane) and the plate was insulated out of plane such that the heating was restricted to two dimensions. 


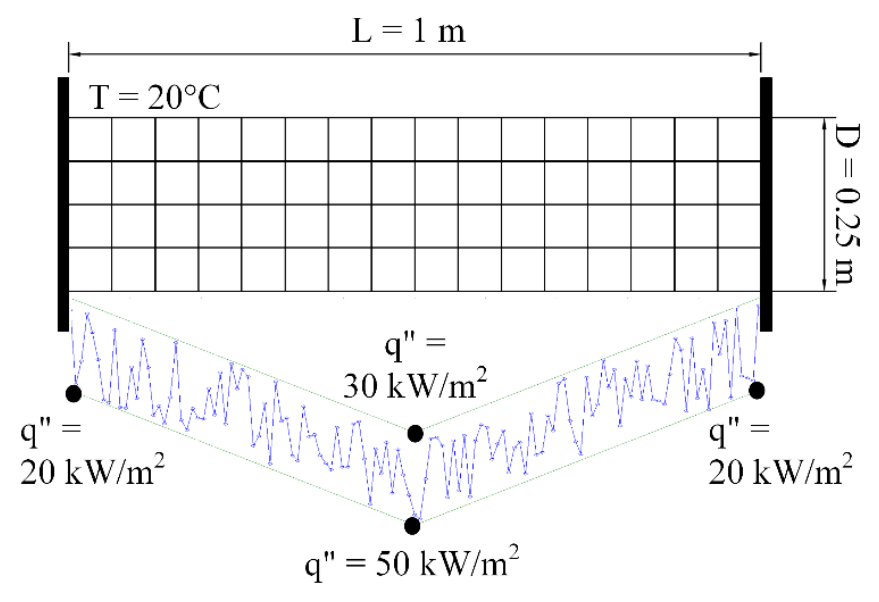

Figure 12: 2D application involving a solid exposed to a random heat flux

The plate was $1 \mathrm{~m}$ long and $0.25 \mathrm{~m}$ deep with a uniform thickness of $0.1 \mathrm{~m}$. The plate was made of steel (specific heat $=465 \mathrm{~J} / \mathrm{kg} \cdot \mathrm{K}$, density $=7,850 \mathrm{~kg} / \mathrm{m}^{3}$, thermal conductivity $=54$ $\mathrm{W} / \mathrm{m} \cdot \mathrm{K})$, and it was assumed that the material properties were independent of temperature for simplicity. The plate had an initial temperature of $20^{\circ} \mathrm{C}$. At the start of the analysis, the bottom edge was instantaneously exposed to the non-uniform net heat flux, while a convective cooling condition was prescribed on the top surface with a heat transfer coefficient of $35 \mathrm{~W} / \mathrm{m}^{2} \cdot \mathrm{K}$ and fluid temperature of $20^{\circ} \mathrm{C}$. It was assumed that the bottom surface was exposed to the nonuniform heat flux and did not interact with the surroundings (i.e., there were no losses due to convection or radiation). A duration of 2,000 seconds was chosen for the analysis.

The analysis was completed using several different mesh configurations to evaluate the accuracy of each method as the mesh was refined. A summary of the mesh configurations is provided in Table 1. The "exact" solution was obtained using a very fine mesh that provided a one-to-one correlation between the heat flux data and the finite element mesh. The results from the exact solution were used to calculate errors in the temperatures associated with each of the homogenization algorithms. 
Table 1: Mesh properties for the 2D case

\begin{tabular}{|c|c|c|c|}
\hline \multirow{2}{*}{ Element Size [m] } & \multicolumn{3}{|c|}{ Number of Elements } \\
\cline { 2 - 4 } & Vertical & Horizontal & Total \\
\hline 0.25 & 1 & 4 & 4 \\
0.125 & 2 & 8 & 16 \\
0.0625 & 4 & 16 & 64 \\
0.03125 & 8 & 32 & 256 \\
0.015625 & 16 & 64 & 1024 \\
\hline
\end{tabular}

The heat flux at the integration points for the averaging, sampling, least squares, and trapezoid methods is plotted in Fig. 13 for mesh configurations of 4 and 1024 elements. The actual heat flux is also plotted for comparison and is represented with a heavier line-weight. It can be seen that the averaging method (Fig. 13a) assumes a uniform heat flux based the average heat flux along the element's length, whereas the sampling (Fig. 13b) and least-squares methods (Fig. 13c) fit the data with a linear function. The trapezoid rule method (Fig. 13d) uses the exact heat flux data in the calculation of the equivalent nodal heat flux vector.

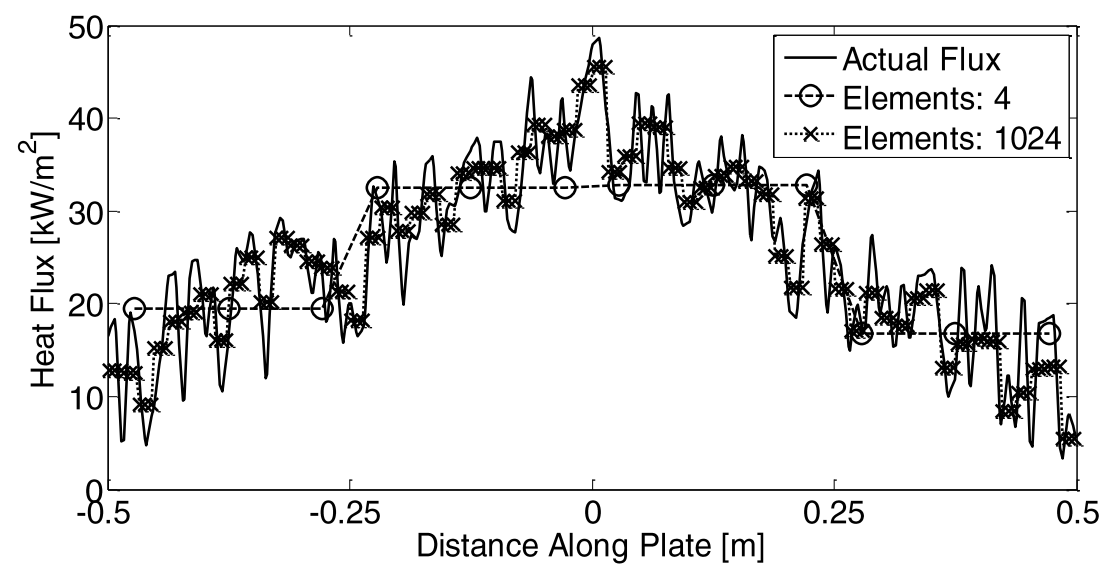

(a) 


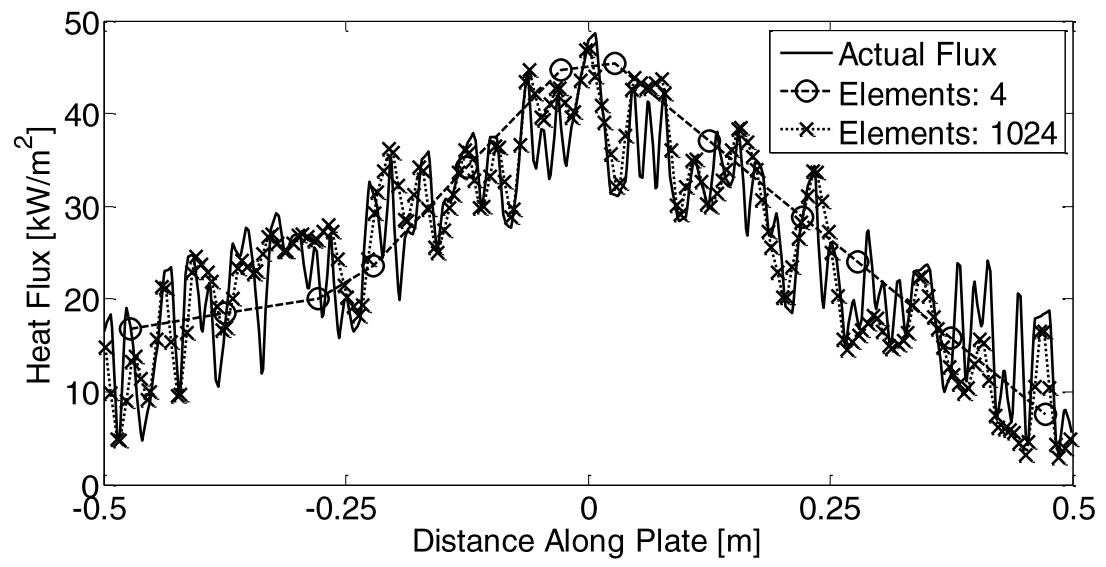

(b)

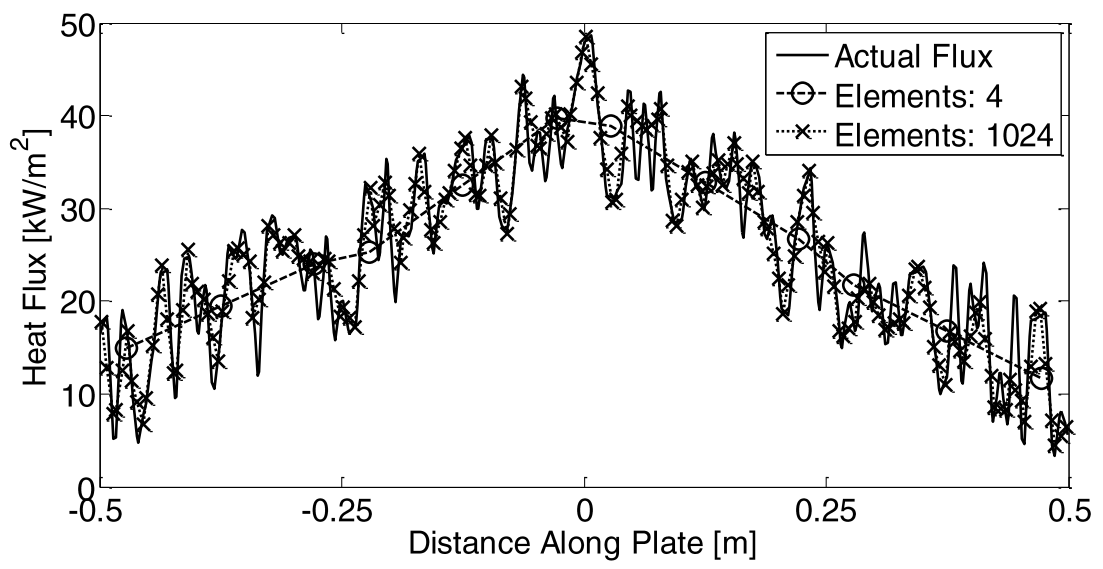

(c)

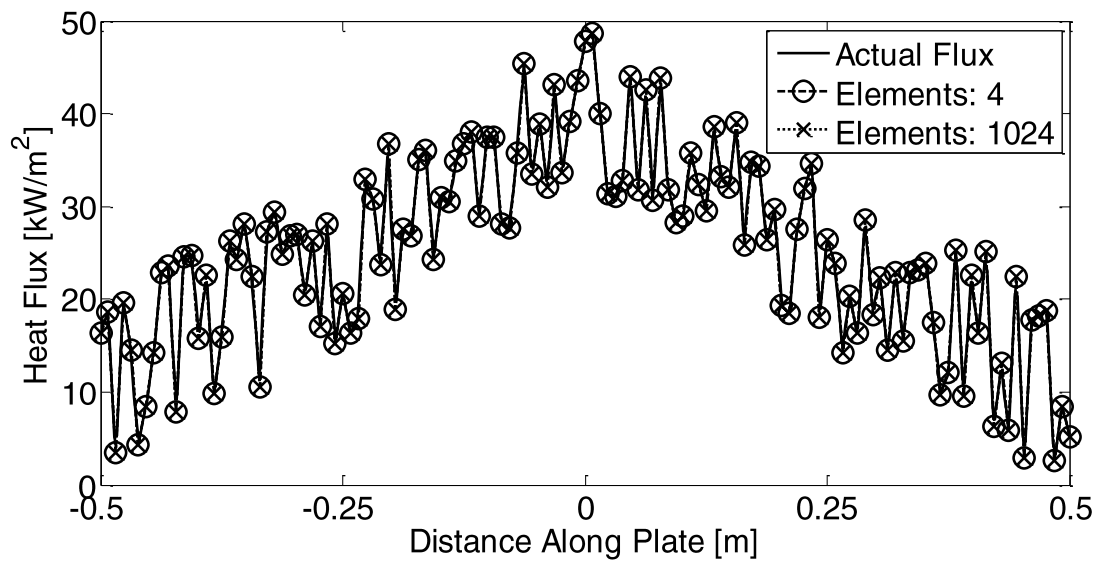

(d)

Figure 13: Heat flux at the integration points: (a) averaging method, (b) sampling method, (c) least squares method, and (d) trapezoid rule 
The approximated heat fluxes shown in Fig. 13 were used in the heat transfer finite element analysis based on Eqs. (4) or (5). The convergence of the solution was investigated based on the mesh configurations given in Table 1 . The nodal temperatures at the heated surface of the plate were calculated for each method and compared to the "exact" (i.e., converged) finite element solution. The "exact" solution was defined as the solution obtained with an element size that corresponded to the spacing of flux data points (i.e., 4,096 elements arranged in a 32x128 grid). Comparisons between the coarsest and finest element meshes are shown in Fig. 14 for each of the methods. Note that the nodal temperatures are shown to be connected by straight lines, although the temperatures are actually interpolated using the quadratic shape functions for the 8node element. From Fig. 14, it can be seen that least-squares (Fig. 14c) and trapezoid rule (Fig. 14d) methods provide a high degree of accuracy for both the coarse and fine finite element meshes, although the least-squares method results in a noticeable difference in temperature prediction at the ends of the plate for the fine mesh. The averaging method gives reasonable results for the fine mesh, although some variations in the calculated temperatures can be seen at the end of the plate (Fig. 14a). The sampling method produces significant differences in the calculated temperatures when compared to the expected values for both the coarse and fine meshes, indicating poor convergence (Fig. 14b). 


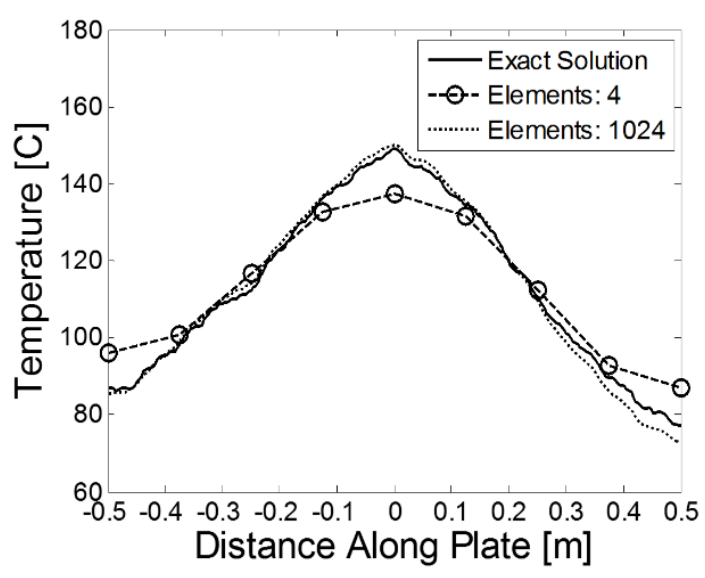

(a)

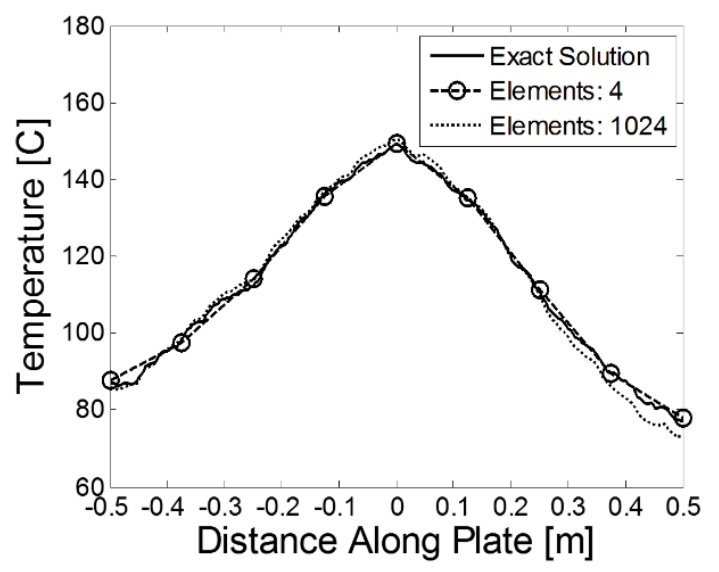

(c)

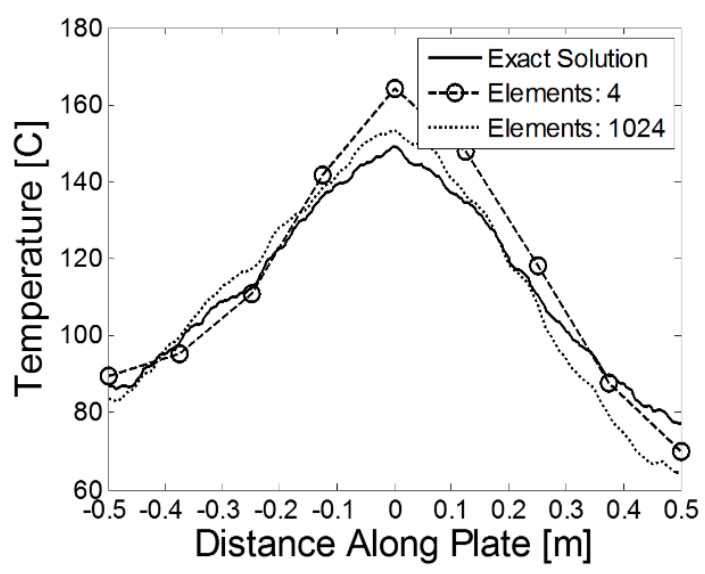

(b)

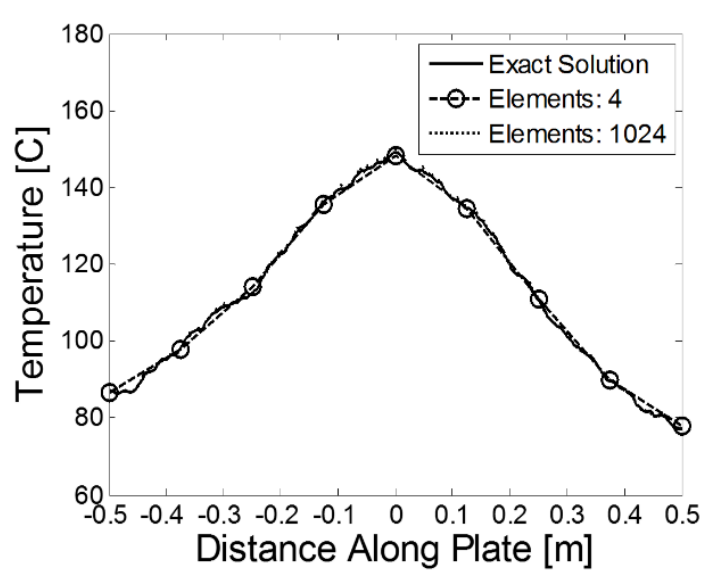

(d)

Figure 14: Heat flux at the integration points: (a) averaging method, (b) sampling method, (c) least squares method, (d) trapezoid rule method

To better gauge the accuracy of the simulation, relative errors were calculated based on the $l^{2}$-norm of the difference in temperature between the coarser element mesh employing the trapezoid rule and the converged finite element solution, as shown in Table 2. The data used for the comparison include all of the nodal temperatures along the heated edge of the plate. In general, the methods tend to converge to the exact solution as the number of elements increases. However, a consistent rate of convergence (i.e., consistently decreasing differences in temperatures between the reference solution and the increasingly fine mesh density) is not attainable with the sampling methods and, to some extent, the least squares methods. The 
increasing value of the norm with mesh refinement that is observed for the least-squares method is caused by a situation in which too few data points exist over an element. The simulation time for each mesh size was comparable regardless of chosen approximation method. Overall, the trapezoid method leads to the smallest relative norm values in all cases and exhibits consistent convergence; it is therefore recommended for spatial homogenization.

Table 2: Vector norm for relative differences in temperature

\begin{tabular}{|l|c|c|c|c|c|}
\hline \multirow{2}{*}{ Method } & \multicolumn{5}{|c|}{ Number of elements } \\
\cline { 2 - 6 } & $\mathbf{4}$ & $\mathbf{1 6}$ & $\mathbf{6 4}$ & $\mathbf{2 5 6}$ & $\mathbf{1 0 2 4}$ \\
\hline Averaging & 4.36 & 1.37 & 1.07 & 1.01 & 1.77 \\
Sampling & 7.14 & 6.75 & 9.52 & 4.73 & 5.32 \\
Least squares & 1.04 & 0.82 & 1.20 & 0.99 & 1.78 \\
Trapezoid rule & 0.85 & 0.59 & 0.41 & 0.24 & 0.26 \\
\hline
\end{tabular}

\subsection{D Application}

To evaluate the performance of the spatial homogenization algorithm based on the trapezoid rule, a 3D application was considered involving a horizontal plate subjected to a localized fire. The plate measured $2 \mathrm{~m} \times 1 \mathrm{~m}$ with a thickness of $5 \mathrm{~cm}$ and had constant thermal material properties, with a specific heat of $1,000 \mathrm{~J} / \mathrm{kg} \cdot \mathrm{K}$, density of $2,000 \mathrm{~kg} / \mathrm{m}^{3}$, and thermal conductivity of $2 \mathrm{~W} / \mathrm{m} \cdot \mathrm{K}$. The plate was located $1 \mathrm{~m}$ above a heptane pool fire, which was characterized by a peak heat release rate of $500 \mathrm{~kW}$ within a fixed burn area of $1,600 \mathrm{~cm}^{2}$. The fire was modeled in FDS with a grid size of $5 \mathrm{~cm}$ for a total duration of 15 minutes. The FDS fire simulation displayed in Fig. 15 features sensors placed at $5 \mathrm{~cm}$ intervals along the top and bottom surfaces of the plate to measure the incident heat flux at each surface, resulting in 800 sensors on each of the top and bottom surfaces of the plate structure. Convection and radiation losses to ambient $\left(T_{\infty}=20^{\circ} \mathrm{C}\right)$ were modeled, with a heat transfer coefficient of $25 \mathrm{~W} / \mathrm{m}^{2} \cdot \mathrm{K}$ and an emissivity of 0.8 . 


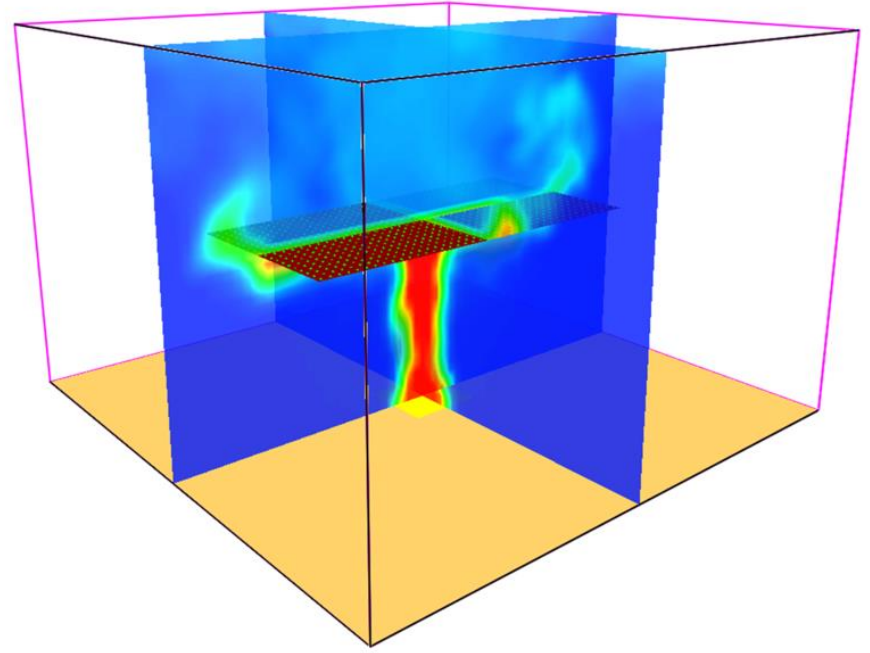

Figure 15: Fire simulation in FDS

The homogenization algorithm was used in conjunction with the 4-node linear shell heat transfer element. A convergence study was performed to measure the performance of the shell element in conjunction with the homogenization algorithm. Four mesh configurations were considered for the plate model: 2x1, 4x2, 8x4, and 16x8, as shown in Fig. 16a. The shell element used five equally spaced layers over the thickness. For comparison, a solid element model was generated in Abaqus using eight-node (linear) brick elements (i.e., DC3D8 elements), as shown in Fig. 16b. Four elements were required through the thickness in order to calculate the temperature gradient through the thickness. An element size of $1.25 \mathrm{~cm} \times 2.5 \mathrm{~cm} \times 2.5 \mathrm{~cm}$ was chosen in order to preserve an appropriate aspect ratio. Thus, the mesh for the solid element model contained 40 elements across the width of the plate, 80 elements along the length of the plate, and 4 elements through the thickness of the plate, for a total of 12,800 elements and 16,605 temperature degrees of freedom. An overview of the mesh details for each of the models is given in Table 3. 

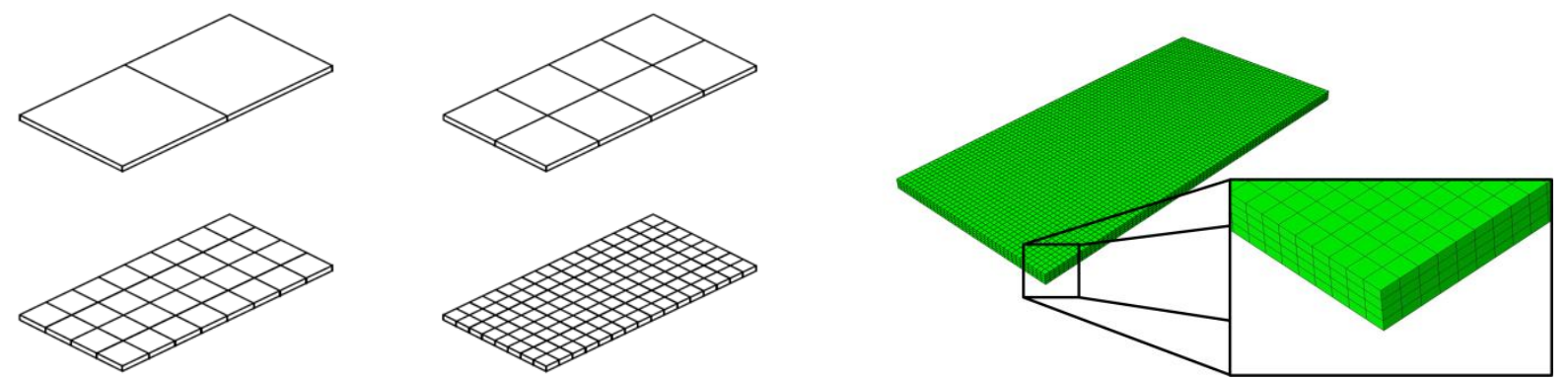

(a)

(b)

Figure 16: Mesh configurations: (a) shell element models of $2 \times 1,4 \times 2,8 \times 4$, and $16 \times 8$ elements, each containing five layers (not depicted); (b) solid element model with four elements through the thickness

Table 3: Mesh properties for the 3D case

\begin{tabular}{c|c|c|c} 
Element Type & Mesh & DOF & Edge Size [cm] \\
\hline Shell UEL & $2 \times 1$ & 30 & 100 \\
Shell UEL & $4 \times 2$ & 75 & 50 \\
Shell UEL & $8 \times 4$ & 225 & 25 \\
Shell UEL & $16 \times 8$ & 765 & 12.5 \\
Solid DC3D8 & $80 \times 40$ & 16,605 & 2.5
\end{tabular}

Surface flux data from the CFD fire simulation in FDS was written to the output file in one-second intervals. Rather than limiting the time step in the solid heat transfer model to one second, the time-averaged subcycling algorithm [4] was used with a time step of 10 seconds to increase the efficiency of the analysis. Subcycling was performed in MATLAB prior to the generation of the input files for the solid heat transfer models. The time-averaged heat flux measured at the sensor in the center of the plate is compared to the actual sensor data from FDS in Fig. 17. After subcycling, the homogenization algorithm based on the trapezoid rule was applied to calculate the equivalent nodal heat fluxes in the shell element models. Homogenization was not needed for the solid element model due to the fact that the mesh in the solid heat transfer model was finer than the CFD mesh. 


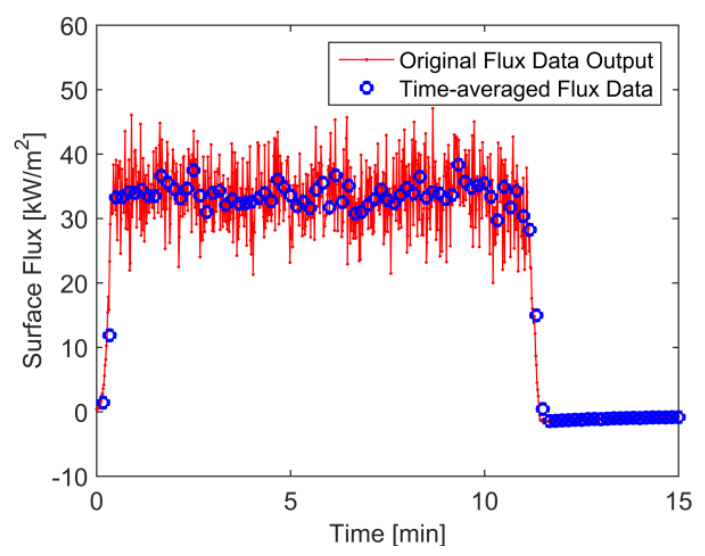

Figure 17: Incident heat flux over time for a sensor at the center of the plate

Accuracy was measured by comparing the shell models to the solid element model. The spatial homogenization algorithm performed well when used with the shell heat transfer elements. Contour plots of the mid-surface temperatures at 12 minutes into the simulation are shown in Fig. 18. Temperatures calculated by the shell models with spatial homogenization are shown in Figs. 18a-18d. Temperatures calculated by the solid element model are shown in Fig. 18e. It can be seen that the shell model converges to the solution calculated by the solid element model. Temperatures are also plotted through the thickness to demonstrate the accuracy of the shell model in predicting cross-sectional temperatures. As illustrated in Fig. 19, a slice through the plate's thickness was taken at the middle of the plate to illustrate the temperature gradients through the thickness. Temperatures are plotted for the solid element model (Fig. 20c) and for the coarsest (2x1) and finest (16x8) shell models (Figs. 20a and b, respectively). Temperature contours are shown at 6 and 12 minutes into the simulation. It can be seen that the shell model converges to the solid element model as the number of elements is increased.

A measure of the relative difference in the computed temperatures at the mid-surface of the plate is provided in the form of the relative $l^{2}$-norm values, as given in Table 4 . The reference solution for computing the norm values was based on the converged solid element model. The 
relative $l^{2}$-norm values were calculated by interpolating between nodal temperatures in the shell models to retrieve the temperatures in the locations of nodes in the solid element model at the mid-surface of the plate. The comparison in Table 4 was prepared using temperatures at the midsurface of the plate only. However, similar convergence properties were exhibited at the top and bottom surfaces of the plate as well.

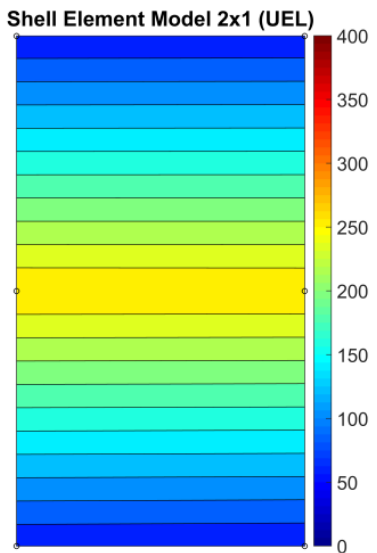

(a)

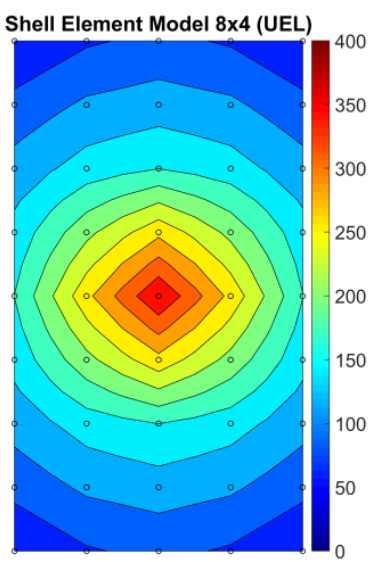

(c)

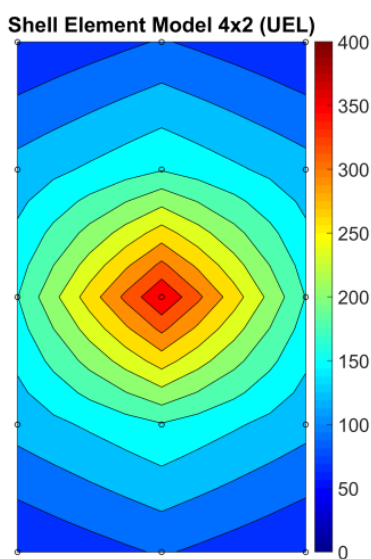

(b)

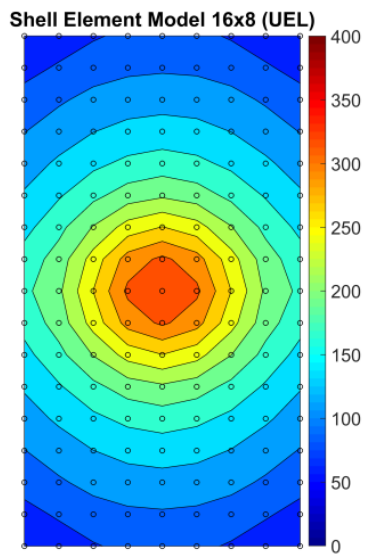

(d) 


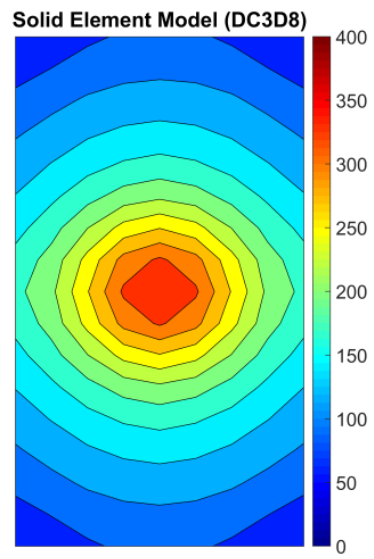

(e)

Figure 18: Contours of the temperature field at the mid-surface of the plate after 12 minutes of fire exposure (a) 2x1 shell model, (b) 4x2 shell model, (c) 8x4 shell model, and (d) 16x8 shell model, and (e) 80x40 solid element model

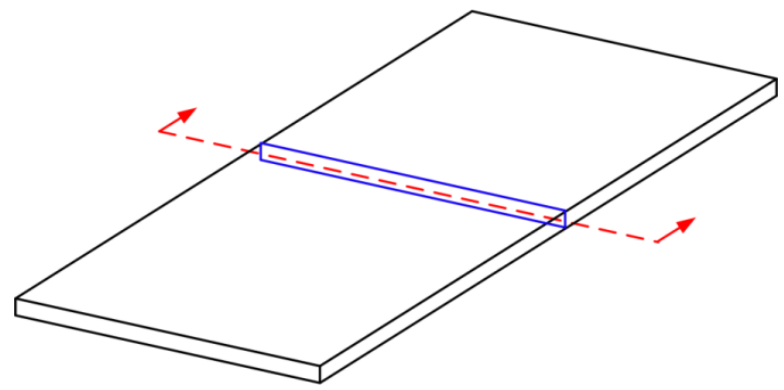

Figure 19: Section for contour plots taken through the thickness
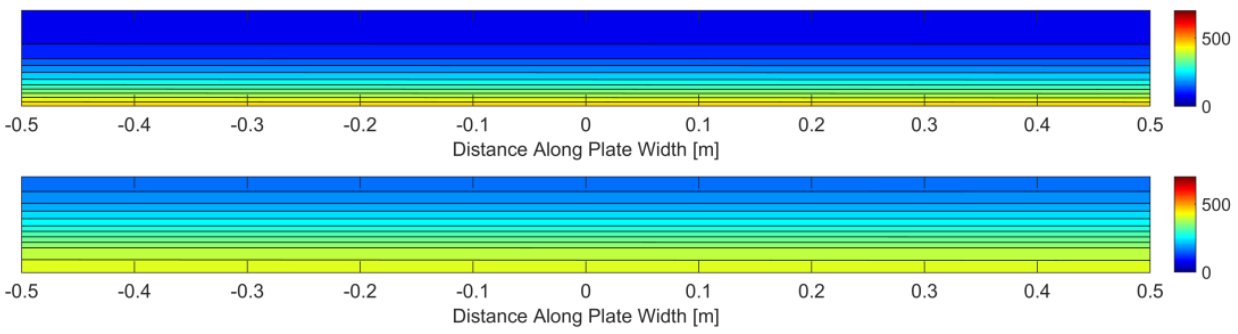

(a)
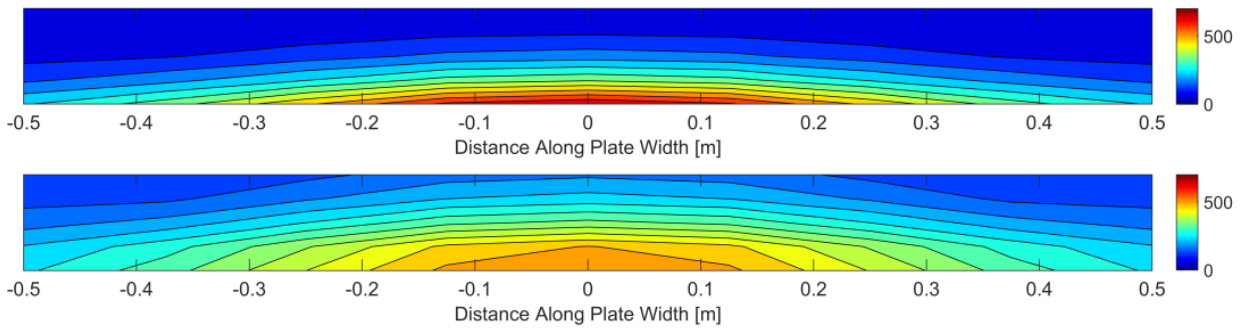

(b) 

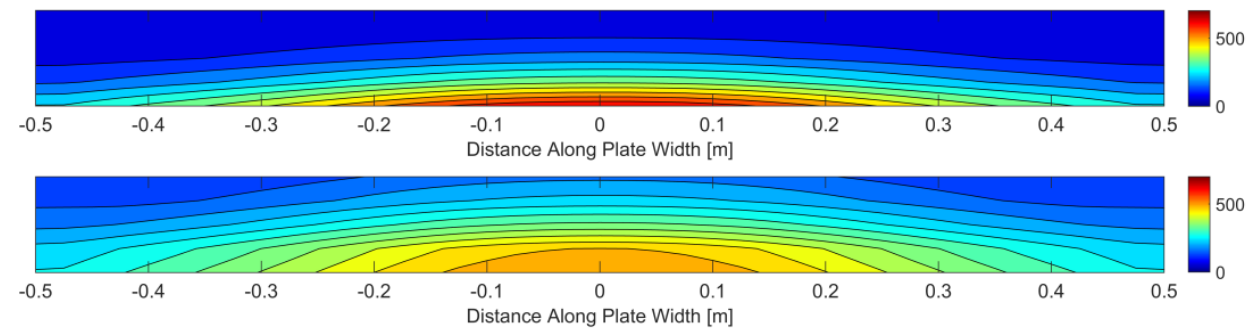

(c)

Figure 20: Temperature field through the thickness at $6 \mathrm{~min}$ (top) and $12 \mathrm{~min}$ (bottom): (a) coarse shell mesh of $2 \times 1$, (b) fine shell mesh of 16x8, and (c) the solid element model

Table 4: Comparison between the shell model and the solid element model

\begin{tabular}{|c|c|c|c|c|c|c|c|}
\hline \multicolumn{3}{|c|}{ Model Details } & \multicolumn{3}{c|}{ Percent Difference (Relative Norm) [\%] } & Computing \\
\cline { 1 - 7 } Element & Mesh & DOF & 3 min & 6 min & 9 min & 12 min & Time \\
\hline Shell UEL & $2 \times 1$ & 30 & 15.6 & 20.2 & 20.3 & 19.7 & $11.5 \mathrm{sec}$ \\
\hline Shell UEL & $4 \times 2$ & 75 & 3.2 & 4.0 & 4.0 & 3.8 & $13.8 \mathrm{sec}$ \\
\hline Shell UEL & $8 \times 4$ & 225 & 3.0 & 3.9 & 3.8 & 3.6 & $20.1 \mathrm{sec}$ \\
\hline Shell UEL & $16 \times 8$ & 765 & 1.2 & 1.5 & 1.5 & 1.4 & $47.4 \mathrm{sec}$ \\
\hline DC3D8 & $80 \times 40$ & 16,605 & -- & -- & -- & -- & $48.5 \mathrm{~min}$ \\
\hline
\end{tabular}

The computing times are also reported in Table 4. The computing time shown in Table 4 includes the subcycling process, the mapping of fluxes to the element surfaces, the computation of equivalent nodal fluxes, and the actual heat transfer analysis. The subcycling process required a mere 0.08 seconds for each model. The time required for the other components of the simulation was directly dependent on the number of degrees of freedom and the numerical integration scheme. As shown in Table 4, the 4x2 shell model employing homogenization by the trapezoid rule was within 4 percent of the solid element model and required only 13.8 seconds to complete the analysis. The $16 \times 8$ shell model provided temperatures that were within 1.5 percent of the solid element model and required 47.4 seconds. For comparison, the solid element model that was used as the reference solution required a total of 48.5 minutes and required significantly more degrees of freedom. 


\section{Conclusions}

A spatial homogenization algorithm was formulated for capturing non-uniform thermal boundary conditions associated with a CFD fire simulation. Energy-equivalent nodal fluxes were calculated for use with macro-level finite elements for heat transfer analysis, which have a coarser mesh in relation to the CFD grid. The method for calculating the nodal fluxes is based on the trapezoid rule for numerical integration. The proposed method was compared to other homogenization techniques including sampling, averaging, and least squares methods for a $2 \mathrm{D}$ application. The trapezoid rule approach offers superior performance because it more closely enforces conservation of energy by accounting for the variations in heat flux over the surface of the structure.

The homogenization algorithm was extended to $3 \mathrm{D}$ analyses and implemented in a macro heat transfer element based on a shell formulation. The homogenization algorithm combined with the shell heat transfer element resulted in an extremely efficient and accurate solution that led to considerable time savings when compared to a solid-element model. Relative errors of less than $1.5 \%$ were reached using 128 layered shell elements in a $16 \times 8$ configuration, requiring less than one minute ( 47.4 seconds) of computing time as opposed to the 48.5 minutes that were needed to complete the solid-element model. This paper does not consider the cost of the CFD fire simulation, which still requires significant computational resources in a coupled firestructure simulation. Additionally, the work described herein only considered a flat rectangular plate in the 3D application and additional work is needed to extend the methodology to tilted and curved geometries.

The applications considered here involved unprotected structures subjected to localized fires with impinging flames. The situation is intended to represent a worst-case scenario in which 
the structure is highly sensitive to variations in surface fluxes and in which the surface fluxes vary considerably over small distances. It is acknowledged that protected structures will be less sensitive to variations in surface fluxes. Additionally, members heated by remote radiation and by optically thick gases may experience less severe fluctuations in surface heat fluxes and therefore may be suitably modeled by other means that assume uniform temperature. In this paper, we advocate for an algorithmically consistent manner for representing the thermal boundary conditions, as achieved by the homogenization algorithm presented here.

\section{Acknowledgements}

This work was supported by the United States Office of Naval Research under contract number N00014-13-C-0373. Any opinions, findings, conclusions, or recommendations are those of the authors and do not necessarily reflect the views of the sponsoring agency.

\section{References}

[1] Eurocode 1: Actions: General actions - Actions on structures exposed to fire, BS EN 19911-2, British Standards Institution, London, 2009.

[2] J. Stern-Gottfried, G. Rein, Travelling fires for structural design - Part I: Literature review, Fire Safety J. 54 (2012) 74-85.

[3] K. McGrattan, H. Baum, W. Mell, R. McDermott, Fire Dynamics Simulator (Version 5) Technical Reference Guide - Volume 1: Mathematical Model, NIST Special Publication 1018-5, National Institute of Standards and Technology, Gaithersburg, MD, 2010.

[4] X. Yu, A.E. Jeffers, A comparison of subcycling algorithms for bridging disparities in temporal scale between the fire and solid domains, Fire Safety J. 59 (2013) 55-61. 
[5] K. Prasad, H. Baum, Federal building and fire safety investigation of the World Trade Center Disaster-Fire structure interface and thermal response of World Trade Center, NIST NCSTAR 1-5G, National Institute of Standards and Technology, Gaithersburg, MD, 2005.

[6] N. Tondini, O. Vassart, J.M. Franssen, Development of an interface between CFD and FE software, in: Fontana, M., Frangi, A., and Knobloch, M. (Eds.), Proceedings of the 7th International Conference on Structures in Fire, ETH Zurich, Zurich, 2012.

[7] N. Tondini, A. Morbioli, O. Vassart, S. Lechene, J.M. Franssen, An integrated modelling strategy between FDS and SAFIR: the analysis of the fire performance of a composite steel-concrete open car park, in: G.Q. Li et al. (Eds.), Proceedings of the $8^{\text {th }}$ International Conference on Structures in Fire, Shanghai, China, 2014.

[8] U. Wickström, D. Duthinh, K. McGrattan, Adiabatic surface temperature for calculating heat transfer to fire exposed structures, Proceedings of the $11^{\text {th }}$ Interflam Fire Science and Engineering Conference, Interscience Communications, London, 2007.

[9] C. Culver, Steel column buckling under thermal gradients, J. Struct. Div. 92 (1972) 18531865.

[10] P. Ossenbruggen, V. Aggarwal, C. Culver, Steel column failure under thermal gradients by member, J. Struct. Div. 99 (1973) 727-739.

[11] J. Kruppa, Some results on the fire behavior of external steel columns, Fire Saf. J. 4 (1981) $247-257$.

[12] J. Witteveen, L. Twilt, A critical view on the results of standard fire resistance tests on steel columns, Fire Saf. J. 4 (1981) 259-270. 
[13] C. Zhang, G.-Q. Li, A. Usmani, Simulating the behavior of restrained steel beams to flame impingement from localized-fires, J. Constr. Steel Res. 83 (2013) 156-165.

[14] L. Chen, C. Luo, J. Lua, FDS and Abaqus coupling toolkit for fire simulation and thermal and mass flow prediction, in: M. Spearpoint (ed.), Proceedings of the IAFSS 10th International Symposium on Fire Safety Science, International Association for Fire Safety Science, UK, 2011.

[15] A.E. Jeffers, E.D. Sotelino, An efficient fiber element approach for the thermo-structural simulation of non-uniformly heated frames, Fire Safety J. 51 (2012) 18-26.

[16] A.E. Jeffers, Heat transfer element for modeling the thermal response of non-uniformly heated plates, Finite Elements in Analysis and Design 63 (2013) 62-68.

[17] A.E. Jeffers, P.A. Beata, Generalized shell heat transfer element for modeling the thermal responses of non-uniformly heated structures, Finite Element in Analysis and Design 83 (2014) 58-67.

[18] A.E. Jeffers, Triangular shell heat transfer element for the thermal analysis of nonuniformly heat structures, J. Struct. Eng. (2015), to appear.

[19] R.D. Cook, D.S. Malkus, M.E. Plesha, R.J. Witt, Concepts and Applications of Finite Element Analysis, $4^{\text {th }}$ ed, John Wiley and Sons, U.S., 2002. 